\title{
Dificultades de la instrucción y el enjuiciamiento de los delitos contra el patrimonio histórico
}

Álvaro Martín, magistrado del Juzgado de lo Penal $n .{ }^{\circ} 2$ de Sevilla

La protección penal del patrimonio histórico se desarrolla conforme a las normas penales que prevén esta cobertura, así como a los procedimientos que tienen por objeto la aplicación de las mismas. A estas cuestiones nos vamos a referir en este artículo, tratando de aproximar nuestro sistema judicial al conocimiento del ciudadano medio e intentando huir de aquellos términos que puedan resultar poco comprensibles.

\section{EL SISTEMA DE PROTECCIÓN PENAL DEL PATRIMONIO HISTÓRICO}

A pesar de lo que comúnmente pueda considerarse, el derecho penal no tiene como finalidad la simple represión o el castigo de las conductas antisociales, sino que el mismo tiende a la protección de los valores esenciales de la sociedad, frente a aquellos comportamientos merecedores de mayor reproche. Es cierto que el mecanismo esencial de este derecho es el punitivo, pero no lo es menos que éste no es más que el instrumento del que se vale para lograr unos fines, que son muy superiores a ese simple castigo como son la protección y en su caso la reparación del bien juridico lesionado.

Desde esta perspectiva la eficacia del derecho penal y del sistema judicial penal ha de medirse con relación al logro de este resultado que puede llegar bien por la vía de la prevención general, término con el que se conoce la disuasión de las conductas delictivas por la advertencia de su castigo; bien por la vía de la prevención especial, la cual se refiere a la disuasión frente a un determinado sujeto (piénsese en la inhabilitación del funcionario que comete un delito en el desempeño de sus funciones); bien por la vía de la restitución del bien lesionado al estado previo a la lesión, lo cual suele operar a través de la responsabilidad civil.

En nuestro Código Penal, en lo referente al patrimonio histórico, se refleja esta visión protectora en los artículos 321 a 324, en los cuales se recogen todos esos mecanismos de protección, sea mediante la sanción a cualquiera que dañe este patrimonio, sea por el castigo del funcionario que favorece este tipo de lesiones, pero, sobre todo, por la llamada constante a la reconstrucción o a la restauración de los bienes destruidos o dañados.
Una vez que hemos fijado cuál es el objeto de la protección penal del patrimonio histórico, debemos estudiar de qué modo se lleva a cabo esta protección a través del sistema penal, cuyo éxito o fracaso en la materia que ahora nos ocupa sólo puede ser establecido bajo el rasero de si consigue el fin que se ha indicado, el de lograr la protección del patrimonio histórico. En la medida en que la respuesta judicial sea rápida, proporcionada y suficientemente disuasoria frente a los ataques a este patrimonio común podremos considerar que alcanza los fines que se le marcan, caso contrario se habrá de concluir con el fracaso de este sistema.

La obtención de estos fines se verifica a través del proceso penal que está inspirado, entre otros muchos principios, por el de presunción de inocencia, con un nivel de exigencia que se va volviendo superior a medida que se avanza en el procedimiento, de modo que si en el inicio la denuncia de hecho, con una mínima base de solvencia, puede ser suficiente para el curso procesal, en las ulteriores fases se irá elevando la necesidad de prueba, hasta alcanzar en la fase de enjuiciamiento el punto álgido, en el que se va a requerir para el dictado de una sentencia condenatoria la prueba plena de los hechos y de la autoría. Esta presunción de inocencia debe ser respetada y valorada en la redacción de todo informe, que debe apartarse de presunciones, legítimas en la esfera administrativa pero que, en el plano penal, estarán avocadas al fracaso por su confrontación con este principio.

Por último, para comprender todo el fenómeno de la protección penal del patrimonio histórico es preciso entender que ésta no es la única que cabe aplicar a este patrimonio. Junto a ellos se encuentra la propia protección administrativa, que también está dotada de instrumentos sancionadores y que puede resultar de aplicación en determinados casos, con exclusión de la respuesta penal.

Se produce asi el encuentro, y en algunos casos la fricción, entre el sistema sancionador administrativo y el sistema penal. No es éste el lugar para estudiar los casos de concurrencia de expediente sancionador administrativo y procedimiento penal, sino el lugar de valorar la coordinación de ambos, para garantizar la eficacia probatoria del primero en este proceso penal, con el objeto de asegurar el buen fin del procedimiento penal y de la propia labor tuitiva de la Administración. 


\section{LA INSTRUCCIÓN}

Como se señala en el título de este artículo, en el proceso penal existen dos fases diferenciadas. La primera es la de instrucción, que no es más que un proceso de investigación sometido a la intervención judicial; la segunda es la de enjuiciamiento, verdadero núcleo de la actuación judicial, pues es en ella donde se ha de abordar la decisión definitiva de toda controversia penal.

En el sistema español la instrucción y enjuiciamiento de los delitos contra el patrimonio histórico viene encomendado a los juzgados de instrucción y a los juzgados de lo penal para cuya determinación existen criterios de atribución de competencia que, de forma sucinta y conforme a lo dispuesto en el artículo 14 de la Ley de enjuiciamiento criminal, podemos resumir en que el Juzgado de Instrucción lo será el del partido en que el delito se hubiese cometido, y el Juzgado de lo Penal el de la circunscripción donde el delito fue cometido (generalmente éstos lo son de circunscripción provincial, aunque existen excepciones a esta regla). A estas reglas básicas se le acompañan otras en el artículo 15 LECrim, que dificilmente serán de aplicación a los delitos que ahora nos ocupan.

Nos centraremos, en primer término, en el estudio de la instrucción de estos delitos, que constituye la fase de mayor dificultad, dada la estructura judicial y la función de investigación tan particular a desempeñar en estos procedimientos.
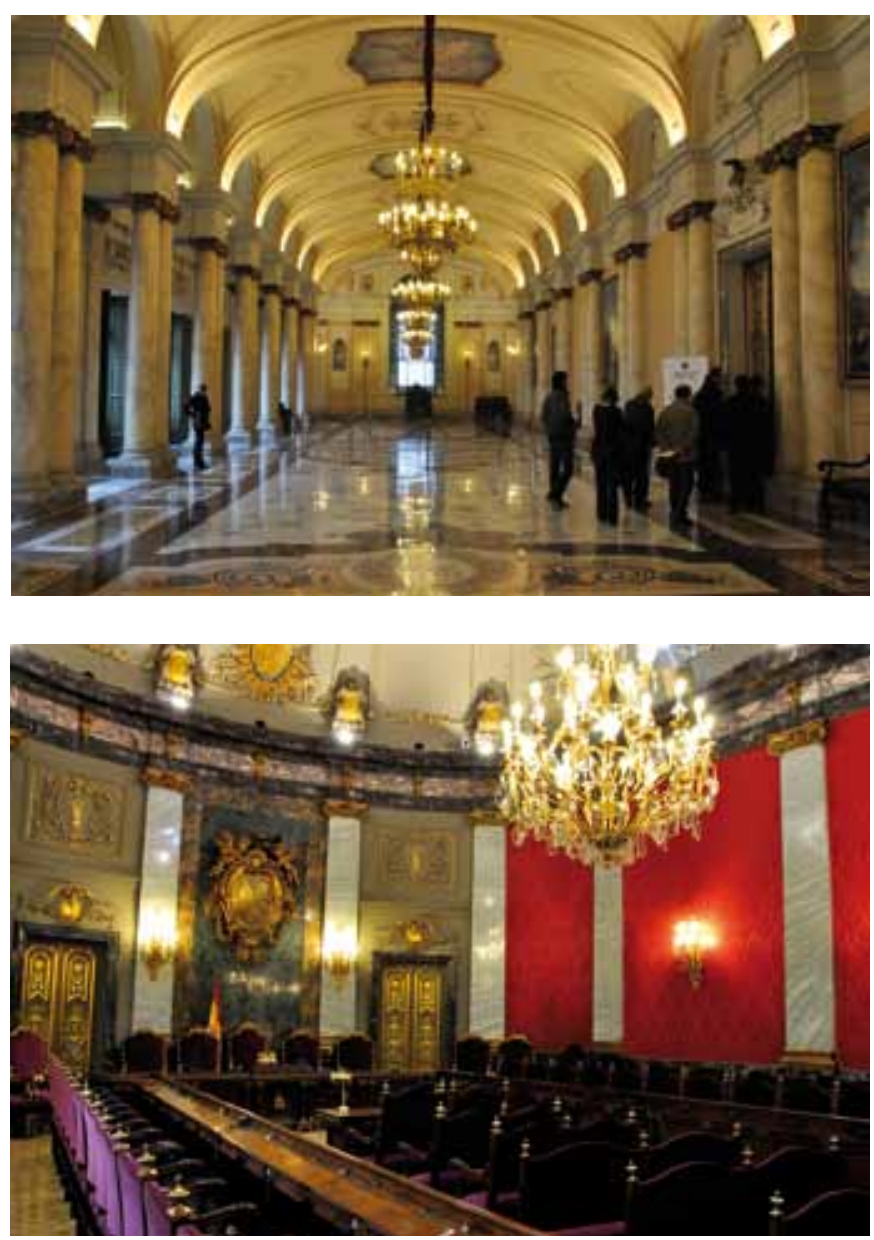

Tribunal Supremo español. Fotos: Luis Sánchez Olmedo
Ya se ha dicho que la competencia para la instrucción corresponde al Juzgado de Instrucción del lugar de comisión del delito, lo cual nos lleva a realizar, primera consideración, la relativa a la ausencia de una especialización en esta materia. A diferencia de lo que puede suceder en el ámbito de la fiscalía, cuya estructura es más flexible que la que se establece en la planta judicial, no cabe especialización de juzgados en la instrucción de estas materias, a pesar de la complejidad técnica que puede llegar a tener la labor a desempeñar.

Esta situación no puede ser superada si se mantienen los actuales criterios de organización de la planta judicial, no pasando de la simple quimera la posibilidad de una especialización como la adoptada en el ámbito de la violencia doméstica, pues ni por volumen de trabajo, ni por demanda social podria llevarse a término. Por otra parte, aunque se lograse, tal especialización no tendria otro alcance que el de asignar en cada partido la instrucción de estos delitos a un determinado juzgado, liberando de este conocimiento a los otros, sin que ello se pudiese traducir en la dotación de un personal especializado, con formación cualificada en esta materia.

La segunda consideración que hemos de hacer, como consecuencia de ese criterio de atribución de competencia territorial, es que en cada partido judicial nos podemos encontrar con distinta respuesta.

Esta afirmación ha de ser entendida en sus justos términos, no se quiere decir que a distinto lugar distinto derecho, sino que a
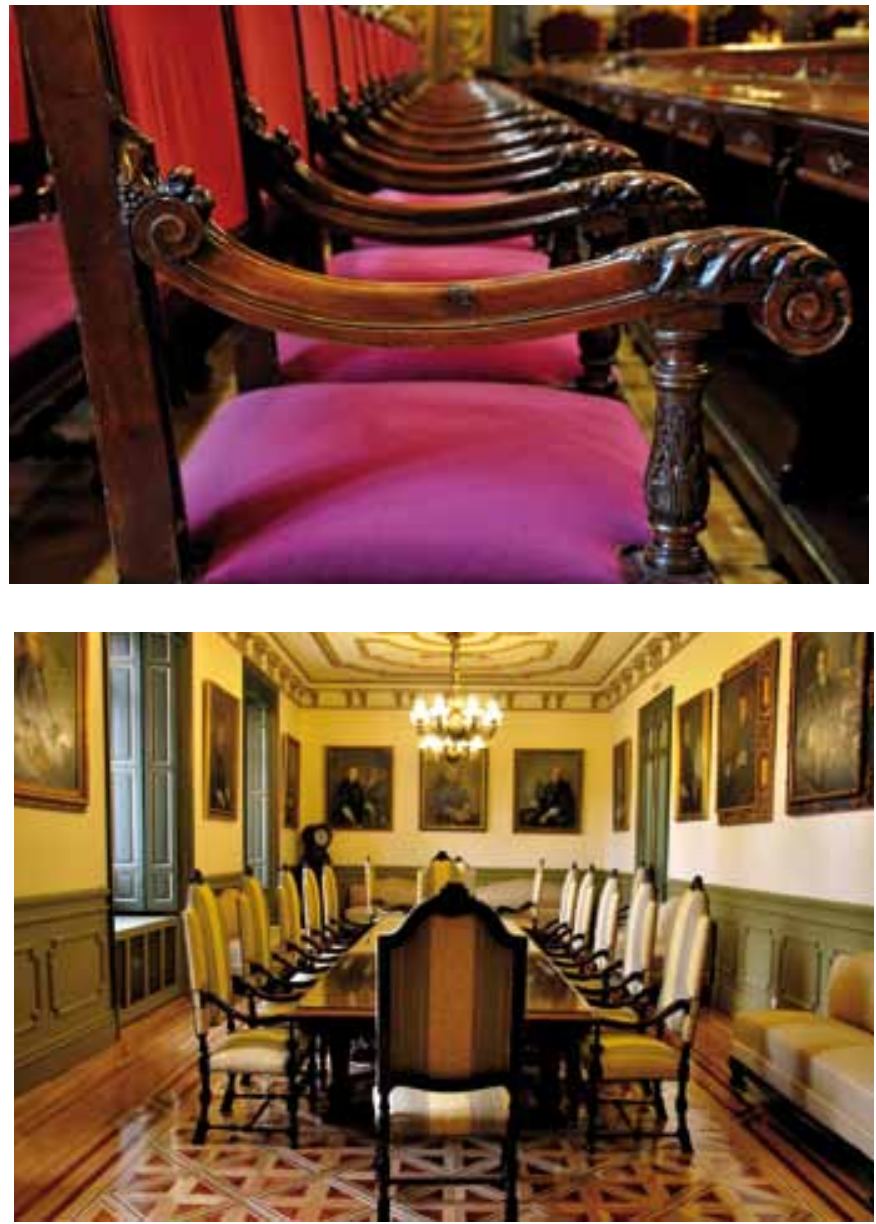
distinto lugar distintos recursos materiales y humanos. Sin lugar a dudas éste es uno de los principales inconvenientes de la actual planta judicial, que avoca a respuestas dispares en cuanto a tiempo y, por qué no decirlo, a eficacia represiva o reparadora. Si pensamos en un Juzgado de Primera Instancia e Instrucción de cualquier pueblo de Andalucia, y lo comparamos con un Juzgado de Instrucción de una de nuestras capitales de provincia, no podremos dejar de notar diferencias sustanciales, tanto en carga de trabajo, como en recursos materiales.

Sin ánimo de ser exhaustivo, podriamos empezar por señalar que, como el propio nombre de los juzgados indica, el juzgado de un pueblo no sólo instruye, sino que también conoce de demandas de orden civil, es decir, ni siquiera se trata de un órgano especializado en la investigación penal. Continuariamos con un somero examen de los recursos humanos de uno y otro juzgado, al menos para destacar que un juzgado de una capital andaluza puede tener el doble de funcionarios destinados a la instrucción que el juzgado de un pueblo y basta atender a la memoria anual del Tribunal Superior de Justicia de Andalucia, Ceuta y Melilla para constatar que, sin embargo, los juzgados de pueblo no tienen una media de asuntos penales que sea inferior a la de los juzgados de instrucción de una capital. Por último, podríamos valorar la experiencia de funcionarios y jueces de unos y otros juzgados, para concluir que el personal de mayor antigüedad se concentra en las capitales de nuestras provincias.

En estas condiciones es claro que la respuesta penal frente a cualquier delito, pero más si se trata de uno de cierta complejidad técnica, se dará con más celeridad en aquellos lugares en los que exista un Juzgado de Instrucción, que en aquellos otros en los que exista uno de Primera Instancia e Instrucción, como también será más eficaz la respuesta de un juzgado de capital de provincia que de cualquier localidad rural.

Ahora bien, no debe pensarse que la dispar respuesta se deba únicamente a la infradotación de los juzgados de primera instancia e instrucción de nuestros pueblos. Cuando tratamos de la protección de patrimonio histórico debemos tener muy presente que la respuesta penal en estos delitos no es espontánea, es decir, no surge de la propia configuración del sistema penal, sino que proviene, como regla general, de la previa labor inspectora de la Administración, que denuncia esta clase de hechos y a los responsables de los mismos.

Cabe plantearse ahora una segunda cuestión relacionada con esta dispar respuesta de nuestro sistema penal, que no es otra que la de si existe la misma estructura administrativa en todas y cada una de nuestras localidades. Como es obvio la respuesta es negativa $y_{1}$ precisamente, es en el ámbito geográfico de mayor debilidad de la instrucción penal donde coincide también una menor implantación de la respuesta administrativa, favoreciendo con ello la aparición de ámbitos de especial vulnerabilidad del patrimonio histórico.

La conjunción de ambas problemáticas es relevante, en primer término porque la ausencia de un buen control administrativo es el presupuesto mismo de la proliferación de los delitos contra el patrimonio histórico, y así en las zonas donde la Administración tenga una más pobre presencia se darán con más facilidad esta clase de hechos; en segundo término porque esa labor administrativa resulta esencial para el ulterior curso del proceso penal, ya que la misma accederá a éste como prueba pericial o documental, y constituirá el núcleo del ulterior debate, de forma que sin un expediente administrativo correctamente formulado, con reflejo claro de los hechos, de por qué se establecen los mismos y de la identidad de sus responsables, difícilmente llegará a producirse la respuesta penal por el daño causado al patrimonio histórico.

Así pues, es en estas zonas en las que se conjugan la más débil implantación administrativa y judicial donde serán más frecuentes este tipo de delitos y, al mismo tiempo, y por ello mismo donde será más difícil su represión.

Constatados estos problemas de la instrucción habria que preguntarse cuáles sean las respuestas que cabe dar a los mismos y analizar si es posible corregir esta situación desde las estructuras actuales, o si no es ello posible y se impone un cambio esencial.

La actual demarcación judicial viene establecida por la Ley $38 / 1988$, de 28 de diciembre, de demarcación y planta judicial, que en sus grandes líneas se limitó a confirmar con algunos matices la distribución de órganos judiciales efectuada a raíz de la antigua Ley provisional sobre organización del poder judicial de 15 de septiembre de 1870 , por lo que arrastra las disfunciones inherentes a unas circunscripciones diseñadas en función de una sociedad radicalmente distinta, fundamentalmente agraria, repartida a lo largo y ancho de la geografía nacional y con una red de comunicaciones inexistente.

Este modelo no es sostenible. La proximidad o cercanía de la justicia no se mide en distancias sino en tiempo. La población se ha trasladado a los polos de desarrollo industrial, abandonando los pueblos, lo que ha provocado que numerosos partidos judiciales, en su día justificados por la situación geográfica y la población, carezcan hoy de sentido.

Es preciso modernizar la demarcación o red judicial conforme a criterios objetivos de población, comunicaciones, servicios a prestar... De la misma forma que no puede existir un hospital o una oficina del Registro de la Propiedad o de la Agencia Tributaria en cada pueblo, tampoco puede pretenderse que el ciudadano tenga un juzgado a la puerta de su casa. Lo que hay que procurar es que exista un juzgado debidamente dotado, con todos los servicios necesarios, a una distancia prudencial que permita tanto el desplazamiento si fuera necesario como, sobre todo, la efectividad del funcionamiento de los juzgados.

Por ello, en las reformas que aparecen en el horizonte se viene a apostar por una nueva demarcación territorial, concentrando los partidos judiciales en sedes que permitan extender a todos los 
La ausencia de un buen control administrativo es el presupuesto mismo de la proliferación de los delitos contra el patrimonio histórico, y así en las zonas donde la Administración tenga una más pobre presencia se darán con más facilidad esta clase de hechos

ciudadanos los servicios que actualmente sólo pueden prestarse en las capitales, dividir o separar las jurisdicciones, implantar servicios comunes generales de registro y reparto, notificaciones y embargos, archivo, ejecución....

Seria en el marco de esta nueva planta judicial donde podrian abordarse las carencias de la instrucción que ya hemos expuesto, de un modo que permitiera superar gran parte de estas limitaciones. No quiere decirse con ello que fuera ése el momento de plantear una especialización en la persecución de este tipo de delitos que, como ya se ha adelantado, no parece justificarse por el volumen de asuntos, pero si que se superarán los problemas de dispar respuesta, acercando la protección a las zonas que actualmente se muestran más vulnerables.

\section{EL ENJUICIAMIENTO}

Otra es la problemática propia de la segunda fase del proceso penal, que afronta el juicio de los hechos que se imputan a una o más personas, frente a las cuales se articula una concreta petición de condena penal y, eventualmente, de responsabilidad civil y cuyo tema principal es el de la prueba.

Es en esta fase donde la exigencia de prueba se vuelve más radical. Ya no se trata de que se denuncie un hecho verosímil, ni de que existan indicios de autoría, ni de que racionalmente se puedan imputar a una o más personas determinadas conductas. Se trata de acreditar que esta o estas personas han cometido unos hechos ciertos.

A nadie se le escapa que los delitos contra el patrimonio histórico, en los que se describen conductas tales como "Los que derriben o alteren gravemente edificios singularmente protegidos por su interés histórico, artístico, cultural o monumental (...) La autoridad o funcionario público que, a sabiendas de su injusticia, haya informado favorablemente proyectos de derribo o alteración de edificios singularmente protegidos (...) la autoridad o funcionario público que por sí mismo o como miembro de un organismo colegiado haya resuelto o votado a favor de su concesión a sabiendas de su injusticia (...) el que cause daños en un archivo, registro, museo, biblioteca, centro docente, gabinete científico, institución análoga o en bienes de valor histórico, artístico, científico, cultu- ral o monumental, así como en yacimientos arqueológicos" son delitos cuya prueba viene asentada, esencialmente, sobre la base de los informes técnicos aportados por quien es competente para ello, que no es otra que la Administración, la cual, además, tiene a su disposición los medios materiales y humanos adecuados para la realización de este tipo de informes, que van a ser traídos al proceso bien como documental, bien como pericial.

Por una parte, el propio concepto de bien protegido avoca a una información por la Administración, que es la competente para acreditar esa cualidad, como lo es para justificar esa alteración grave del bien, o su daño.

Por otra, el mero hecho de que se contemple la reconstrucción o restauración del bien vuelve a avocar a la actuación de la Administración, para concretar los mecanismos de reparación, en tanto en cuanto ello es algo ajeno al conocimiento del órgano judicial y precisa de su concreción por parte de la Administración.

El que el informe técnico sea la piedra angular de este tipo de procedimientos no debe llevar a considerar que el mismo se respete en su integridad, conforme a las presunciones válidas en el derecho administrativo, y sin mayor contraste. Por el contrario, su carácter de elemento esencial lo torna en eje de la discusión penal, y por ello la defensa está legitimada para plantear controversia sobre el mismo, sea por la vía de periciales contradictorias, sea por la vía de discutir su tenor.

En este punto cobra gran trascendencia la correcta preparación del informe por el técnico que lo redacta, el cual debe tener presente las propias reglas del derecho penal, en el que no cabe presumir dato alguno, más allá de aquellos hechos notorios que están exentos de prueba. Esa correcta preparación también debe traducirse en una adecuada explicación de sus conclusiones, y de los datos que le han llevado a las mismas, a verificar en el acto del juicio, donde podrán ser cuestionados las unas y los otros.

En definitiva, todo aquel que redacta un informe avocado a un procedimiento penal debe ser consciente de que ese informe puede ser sometido a severa contradicción y que, por tanto, su labor no termina al realizar unas conclusiones fundadas en criterios administrativos, sino que abarca la defensa de las mismas de acuerdo al sistema de garantías penales.

\section{Bibliografía}

AA. W. (1996) La prueba en el proceso penal (2 vol.) Madrid: Consejo General del Poder Judicial, 1996 (Cuadernos de Derecho Judicial)

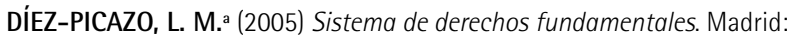
Editorial Thomson, Civitas, 2005 LUZÓN CUESTA, J. M. a (1996) Compendio de derecho penal: Parte general y especial. S. I.: Editorial Dykinson S.L., 1996

MUÑOZ CONDE, F. (2001) Teoría General del delito. Bogotá: Editorial Temis, 2001 